\title{
Visual Suppression in Intermittent Exotropia during Binocular Alignment
}

\author{
Ignacio Serrano-Pedraza, ${ }^{1}$ Vina Manjunath, ${ }^{2}$ Olaoluwakitan Osunkunle, ${ }^{3}$ \\ Michael P. Clarke, ${ }^{2}$ and Jenny C. A. Read ${ }^{1}$
}

Purpose. To investigate the cortical mechanisms that prevent diplopia in intermittent exotropia $(\mathrm{X}(\mathrm{T}))$ during binocular alignment (orthotropia).

Methods. The authors studied $12 \mathrm{X}(\mathrm{T})$ patients aged 5 to 22 years. Seventy-five percent had functional stereo vision with stereoacuity similar to that of 12 age-matched controls $(0.2-3.7$ min arc). Identical face images were presented to the two eyes for $400 \mathrm{~ms}$. In one eye, the face was presented at the fovea; in the other, offset along the horizontal axis with up to $12^{\circ}$ eccentricity. The task was to indicate whether one or two faces were perceived

REsults. All X(T) patients showed normal diplopia when the nonfoveal face was presented to nasal hemiretina, though with a slightly larger fusional range than age-matched controls. However, 10 of 12 patients never experienced diplopia when the nonfoveal face was presented to temporal hemiretina (i.e., when the stimulus simulated exodeviation). Patients showed considerable variability when the single image was perceived. Some patients suppressed the temporal stimulus regardless of which eye viewed it, whereas others suppressed a particular eye even when it viewed the foveal stimulus. In two patients, the simulated exodeviation might have triggered a shift from normal to anomalous retinal correspondence.

Conclusions. Antidiplopic mechanisms in $\mathrm{X}(\mathrm{T})$ can be reliably triggered by purely retinal information during orthotropia, but the nature of these mechanisms varies between patients. (Invest Ophthalmol Vis Sci. 2011;52:2352-2364) DOI:10.1167/ iovs.10-6144

Thtermittent exotropia $(\mathrm{X}(\mathrm{T}))$, which has a prevalence $1>0.5 \%$, is the most common form of exotropia. ${ }^{1}$ In $\mathrm{X}(\mathrm{T})$ patients, one eye intermittently deviates outward, most often when the patient is tired, ill, under stress, or in particular test situations. ${ }^{2}$ Interestingly, when one eye is deviated, despite the fact that images then fall in noncorresponding points of the two retinas, $\mathrm{X}(\mathrm{T})$ patients do not experience diplopia; often they are not even aware of the exodeviation. ${ }^{2-4}$ This suggests

From the ${ }^{1}$ Institute of Neuroscience, Faculty of Medical Sciences, Newcastle University, Newcastle upon Tyne, United Kingdom; ${ }^{2}$ Eye Department, Royal Victoria Infirmary, Newcastle upon Tyne, United Kingdom; and ${ }^{3}$ Gonville and Caius College, University of Cambridge Cambridge, United Kingdom.

Supported by Royal Society University Research Fellowship UF041260 (JCAR) and Medical Research Council New Investigator Award 80154 (JCAR)

Submitted for publication June 30, 2010; revised August 21 and October 11 and 29, 2010; accepted November 11, 2010.

Disclosure: I. Serrano-Pedraza, None; V. Manjunath, None; $\mathbf{O}$ Osunkunle, None; M.P. Clarke, None; J.C.A. Read, None

Corresponding author: Ignacio Serrano-Pedraza, Institute of Neuroscience, Faculty of Medical Sciences, Newcastle University, Newcastle upon Tyne, NE2 4HH, UK; i.s.pedraza@ncl.ac.uk. that these patients have developed mechanisms to avoid the diplopia that acute exodeviation causes in persons with normal visual experience.

Studies of $\mathrm{X}(\mathrm{T})$ patients during deviation have indicated that these antidiplopic mechanisms usually involve interocular suppression, ${ }^{5-9}$ in which information from one eye is prevented from reaching consciousness The shape, size, and location of suppression scotomata appear to depend strongly on the experimental stimuli, ${ }^{6,9,10}$ but they generally lie in the temporal hemiretina of the deviating eye. Anomalous retinal correspondence, a remapping of the usual relationship between the two eyes, has also been proposed. ${ }^{6,10,11}$

Almost all these studies have examined $\mathrm{X}(\mathrm{T})$ patients during periods of exodeviation using techniques such as occlusion to induce the exodeviation. Only two studies ${ }^{7,9}$ have studied single vision during fixation. Thus, exactly what triggers suppression in intermittent exotropia remains unclear. It could be that suppression is triggered by the eye movement itself, such as by proprioceptive information, indicating that exodeviation has occurred. Alternatively, suppression could be triggered directly from the retinal images. For example, if the two retinal images are identical apart from a large horizontal offset, this could be taken as retinal evidence that exodeviation has occurred. In the present work, we examined this question by quantitatively measuring single versus double vision in 12 patients between 5 and 22 years of age with diagnoses of intermittent exotropia.

\section{General Methods}

Methods specific to each experiment are given in that section.

\section{Participants}

Patients were recruited at the Eye Clinic, Royal Victoria Infirmary, Newcastle on Tyne, United Kingdom, by MPC and VM. Most recruits were children because $\mathrm{X}(\mathrm{T})$ patients tend to have ceased attending the clinic by adulthood. Patients with convergence insufficiency (near angle of exotropia greater than distance angle), constant exotropia, or significant coexisting ocular abnormality, such as cataract, were excluded. We recruited $12 \mathrm{X}(\mathrm{T})$ patients between 5 and 22 years of age (Table 1) and 12 age-matched control participants between 3.5 and 20 years of age (Table 2). All 24 patients and control participants performed experiment 1 , a measure of stereoacuity. In assessing the variation in stereoacuity within the control population, we also used an existing data set within our laboratory containing stereoacuity for an additional 10 controls from 3.5 to 29 years of age, measured with the same equipment and procedure. All 24 patients and control participants performed experiment 2. A subset of eight patients also performed experiment 3; this experiment was designed to probe the nature of the antidiplopic mechanism and, hence, could not be performed by controls.

All participants, controls, and patients had normal or corrected-tonormal refraction and viewed the screen with natural pupils through 
Table 1. Clinical Details for Patient Participants

\begin{tabular}{|c|c|c|c|c|c|c|c|c|c|}
\hline \multirow{2}{*}{$\begin{array}{c}\mathbf{X}(\mathbf{T}) \\
\text { Patients }\end{array}$} & \multirow{2}{*}{$\begin{array}{c}\text { Age at } \\
\text { Testing (y) }\end{array}$} & \multirow[b]{2}{*}{ Sex } & \multicolumn{2}{|c|}{$\begin{array}{c}\text { Visual Acuity } \\
\text { (logMAR* Score) }\end{array}$} & \multirow{2}{*}{$\begin{array}{l}\text { Interocular } \\
\text { Acuity } \\
\text { Difference }\end{array}$} & \multirow{2}{*}{$\begin{array}{l}\text { Eye That } \\
\text { Deviates }\end{array}$} & \multicolumn{2}{|c|}{$\begin{array}{c}\text { Angle of } \\
\text { Deviation (Prism } \\
\text { Diopters) Where } \\
\text { Available }\end{array}$} & \multirow{2}{*}{$\begin{array}{c}\text { Age at } \\
\text { Diagnosis }\end{array}$} \\
\hline & & & Left Eye & Right Eye & & & Near & Distance & \\
\hline X01 & 11 & Female & 0.15 & 0.2 & 0.05 & Right & 6 & 30 & $6.5 \mathrm{y}$ \\
\hline X02 & 9 & Male & 0.000 & 0.000 & 0.000 & Right & - & - & $4 y$ \\
\hline X05 & 22 & Male & -0.100 & -0.200 & 0.100 & Left & - & - & $4 y$ \\
\hline X06 & 7 & Female & 0.025 & 0.500 & 0.475 & Right & - & 35 & $2 y$ \\
\hline X07 & 7 & Female & 0.000 & 0.000 & 0.000 & Left & - & 18 & $1 \mathrm{y}$ \\
\hline X08 & 6 & Female & 0.000 & 0.000 & 0.000 & Left & - & - & $1 \mathrm{y}$ \\
\hline X09 & 9 & Male & 0.025 & 0.000 & 0.025 & Right & - & 10 & $5 y$ \\
\hline $\mathrm{X} 10$ & 7 & Male & 0.225 & 0.175 & 0.050 & Left & - & 25 & $10 \mathrm{mo}$ \\
\hline
\end{tabular}

${ }^{*}$ logMAR crowded test (Keeler limited).

polarizing filters. Their visual acuity is given in Tables 1 and 2 . It was in the normal range $( \pm 0.2 \log$ MAR $)$ for both eyes in all control participants except the youngest, aged 3, from whom we recorded a value of 0.27 in one eye. Patient participants all had normal acuity in the habitually fixating eye; two participants had acuity worse than +0.2 logMAR in the habitually deviating eye. The study conformed to the Declaration of Helsinki and was approved by Newcastle University's Human Psychology Ethics Committee and the UK National Health Service National Research Ethics Service (County Durham and Tees Valley 1 Research Ethics Committee [REC]). All participants, and parents of child participants, were provided with an age-appropriate printed information sheet, approved by the NHS REC, and were given the opportunity to ask further questions before agreeing to participate. Written consent was obtained from all adult participants and from a parent of each child participant. Where possible, written assent was also obtained from child participants. All the experiments were carried out in the stereovision laboratory of JCAR at the Institute of Neuroscience of Newcastle University.

\section{Stimulus Presentation}

The experiments were carried out in a laboratory in dim ambient lighting. Stimuli were presented on a rear projection screen, frontoparallel to the observers, who viewed it from a distance of $120 \mathrm{~cm}$.
Equipment details were as given previously, ${ }^{12}$ except that the projected image measured $71 \times 53 \mathrm{~cm}$ subtending $33^{\circ} \times 25^{\circ}$. Stimuli were generated in MATLAB 7.5 (R2007b) with the Psychophysics Toolbox extensions ${ }^{13,14}$ (www.psychtoolbox.org). On our display, white had a luminance of $4 \mathrm{~cd} / \mathrm{m}^{2}$, reduced to $2.8 \mathrm{~cd} / \mathrm{m}^{2}$ when viewed through the polarizing glasses, and black had a luminance of $0.07 \mathrm{~cd} / \mathrm{m}^{2}$, reduced to $0.05 \mathrm{~cd} / \mathrm{m}^{2}$.

To make the experiments as pleasant as possible for children, the laboratory was furnished with colorful nursery wall hangings and rugs. Participants sat at a brightly colored table and indicated their responses by hitting large, brightly colored response buttons (AbleNet, Roseville, MN; www.ablenetinc.com). We found in pilot experiments that child participants struggled with a two-alternative forced-choice paradigm because they were reluctant to guess. As a result, we included a "don't know" button. To ensure our young participants remembered the correct meaning of the three buttons, each button press triggered a recording of a child's voice saying the meaning of that button. The meaning of the buttons was also indicated visually throughout the experiment; for example, for the stereoacuity task, solid models depicting the front and back surfaces were placed near the appropriate button. On random trials, the computer also played a recording of a child's voice giving encouragement (e.g., "You're very good at this!"). This encouragement was given, on average, at every fifth trial, and only

TABLe 2. Visual Acuity Measurements for Control Participants

\begin{tabular}{|c|c|c|c|c|c|}
\hline \multirow[b]{2}{*}{ Controls } & \multirow[b]{2}{*}{ Age at Testing (y) } & \multirow[b]{2}{*}{ Sex } & \multicolumn{2}{|c|}{$\begin{array}{c}\text { Visual Acuity } \\
\text { (logMAR* Score) }\end{array}$} & \multirow[b]{2}{*}{ Difference } \\
\hline & & & Left Eye & Right Eye & \\
\hline $\mathrm{C} 01$ & 5 & Female & -0.1 & -0.1 & 0 \\
\hline $\mathrm{CO} 2$ & 5 & Female & -0.1 & -0.1 & 0 \\
\hline $\mathrm{CO3}$ & 7 & Male & -0.3 & -0.3 & 0 \\
\hline $\mathrm{C} 04$ & 3.5 & Female & 0.27 & 0.075 & 0.195 \\
\hline $\mathrm{C} 05$ & 4 & Male & 0.125 & 0.1 & 0.025 \\
\hline $\mathrm{C} 06$ & 5 & Male & 0.2 & 0.1 & 0.1 \\
\hline $\mathrm{C} 07$ & 16 & Male & -0.1 & -0.1 & 0 \\
\hline $\mathrm{C} 08$ & 7 & Male & Not tested & Not tested & Not tested \\
\hline $\mathrm{CO9}$ & 8 & Male & 0 & -0.05 & 0.05 \\
\hline $\mathrm{C} 10$ & 6 & Female & 0.05 & 0 & 0.05 \\
\hline C11 & 11 & Female & Not tested & Not tested & Not tested \\
\hline $\mathrm{C} 12$ & 20 & Female & Not tested & Not tested & Not tested \\
\hline
\end{tabular}

* logMAR crowded test (Keeler limited). 
on trials in which the participant's response was correct, to avoid reinforcing an incorrect response. Because it was given so sparsely, it did not provide reliable feedback about correctness.

The tasks were explained using presentation software (PowerPoint; Microsoft, Redmond, WA) suitable for young children and by completing a few example trials. Child participants generally completed the experiments in blocks of 5 minutes, interspersed with breaks for rest and refreshment. On average, each experiment required 10 to 15 minutes of testing time.

\section{Experiment 1: Stereoacuity Measurement}

\section{Specific Methods}

Stereoacuity was measured using a random-dot stereogram depicting a disparate disc, $8^{\circ}$ in diameter, on a zero-disparity background. The random-dot patterns consisted of circular white dots, $0.1^{\circ}$ in diameter, distributed uniformly and randomly across a black background with a density of $20 \mathrm{dots} / \mathrm{deg}^{2}$. The random-dot pattern occupied a square $23.86^{\circ} \times 23.86^{\circ}$; the remainder of the screen was black. All dots had zero disparity apart from those within $4^{\circ}$ of the center, which were all given the same disparity, chosen according to a Bayesian staircase procedure. Subpixel disparities were achieved with anti-aliasing. Participants were asked to report, by means of button press, whether the disparate disc appeared in front of or behind the background. If the participant pressed "don't know," the computer randomly allocated this as either "in front" or "behind" with equal probability. This might have resulted in higher threshold estimates; therefore, where possible, participants were encouraged to make a guess rather than use the "don't know" option. Participants could view stimuli as long as required before making their response, so the experiment proceeded at a pace determined by the observer. Between trials, a small fixation cross $\left(0.47^{\circ} \times 0.47^{\circ}\right)$ in the center of the screen, flanked by vertical and horizontal Nonius lines of length $0.6^{\circ}$, was presented for $500 \mathrm{~ms}$

Before starting the experiment, participants were familiarized with the task by completing a few "easy" example trials, on which the disparity was set to be well above threshold (typically, $7 \mathrm{~min}$ arc). Disparity thresholds were measured using an adaptive Bayesian staircase $\mathrm{e}^{15}$ in a front of or behind the discrimination task. In each trial, the sign of the disparity was selected randomly. In $90 \%$ of trials, the magnitude of disparity was given by the adaptive staircase. In a randomly interleaved $10 \%$ of trials, the magnitude of disparity was set to a large value known to be easily visible to the participant. We have found these "easy" trials very helpful in maintaining the motivation of young or inexperienced participants, especially when disparity gets close to the threshold at the end of the staircases. These easy trials did not form part of the staircase and were not used in the threshold estimation. Trials on which the participant pressed "don't know" did form part of the threshold estimation, using the response assigned by the computer. Characteristics of the Bayesian staircases were as follows: the previous probability density function was uniform ${ }^{15,16}$; the model likelihood function was the logistic function adapted from Appendix A of García-Pérez, ${ }^{17}$ with a spread value of 0.8 (with delta parameter equal to 0.01), a lapse rate of 0.01 , and a guess rate of 0.5 ; the value of the disparity in each trial was obtained from the mean of the posterior probability distribution ${ }^{18}$; the final threshold was estimated from the mean of the final probability density function; and the stopping rule for the staircases was the number of trials ${ }^{15,19}$ (in particular, 50 trials were used). In total, two threshold estimates were obtained for each participant, each taking approximately 7 minutes to be obtained.

\section{Results}

Figure 1 shows measured stereoacuity for 22 controls (open circles and squares), including the 12 control participants who performed experiment 2 (open circles), and for the $12 \mathrm{X}(\mathrm{T})$ patients (red circles). Stereoacuity is expressed as the threshold, in minutes of arc, for a performance rate of $85 \%$ correct. On average, both control and patient participants pressed the "don't know" button on $10 \%$ of trials, primarily those at or below threshold. There was no difference in the frequency of "don't know" responses between the two groups $(P=0.5$, two-sample $t$-test). Measured stereoacuity improves (i.e., threshold decreases) with age, reaching a plateau between 15 and 20 years of age. It is obviously possible that some of the measured improvement in stereoacuity up to age 15 in fact reflected improvement in concentration/motivation; especially for the youngest participants, our measurements must be regarded as lower bounds.

However, the main purpose of Figure 1 is to enable a comparison between the stereoacuity of $\mathrm{X}(\mathrm{T})$ patients (red symbols) and of controls (open symbols). To quantify this while allowing for variation due to age, we used the black line in Figure 1, which was fitted to data from all 22 controls. We defined the "normal range" of stereoacuity as $\pm 2 \mathrm{SD}$, corresponding to the $95 \%$ confidence interval for a Gaussian distribution. All 12 of our control participants (open circles) and 9 of 12 of the $\mathrm{X}(\mathrm{T})$ patients (red circles) are within this ageadjusted normal range, whereas the remaining $3 \mathrm{X}(\mathrm{T})$ patients have clearly poorer stereoacuity. Thus, despite their eye-movement disorder, all our $\mathrm{X}(\mathrm{T})$ patients have functional stereo

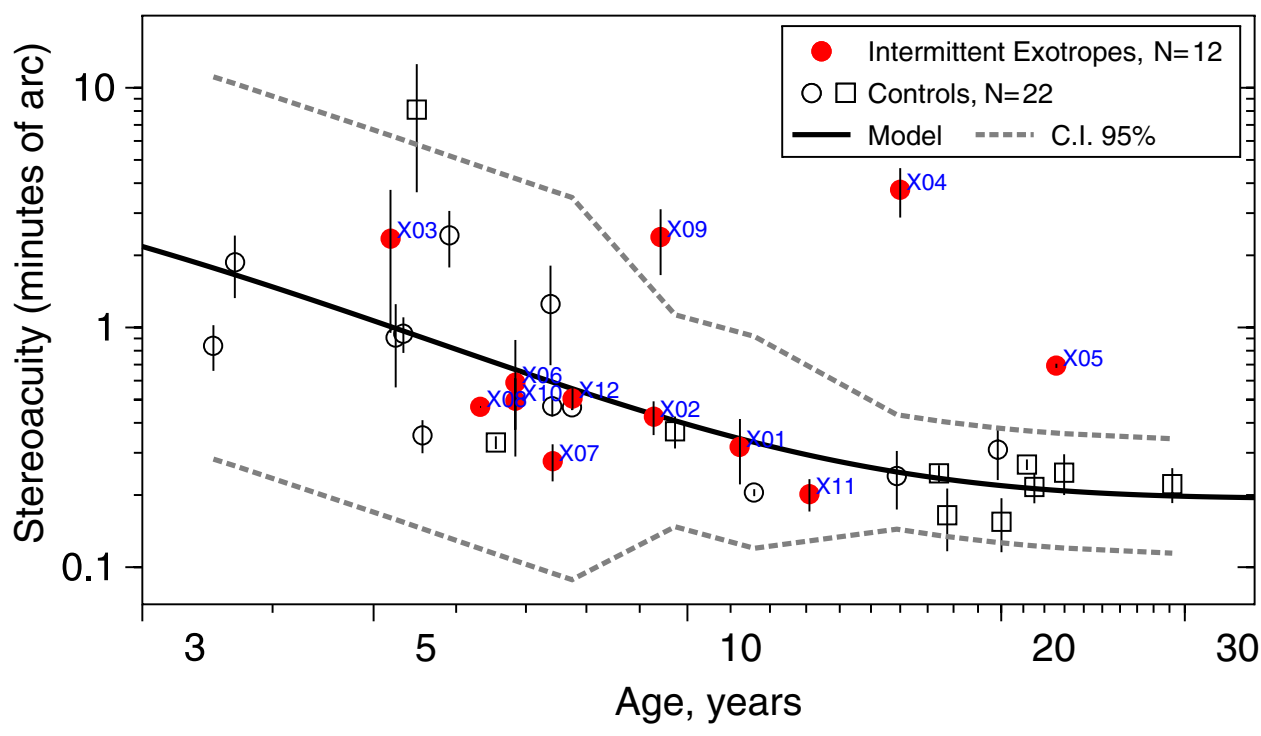

FIgURE 1. Stereoacuity as a function of age for 22 controls (open circles: results for the 12 control participants who performed experiment 2; open squares: results for an additional 10 controls) and $12 \mathrm{X}(\mathrm{T})$ patients (red circles). Symbols represent the mean and error bars \pm the SEM of the stereoacuity thresholds. The black line is a model fitted to the control data. We fitted the equation Stereoacuity (Age) $=60 \times 10^{\left[\mathrm{B}-\mathrm{b} \times \exp (-\mathrm{Age})^{\mathrm{A}}\right]}$ to the control stereoacuity thresholds (in minutes of arc) using the least-squares method. The fitted values of the three parameters were $\mathrm{B}=-2.49 ; \mathrm{b}=$ $-1.78 ; \mathrm{a}=0.17$. Dotted lines: bounds of the estimated $95 \%$ confidence interval. 
vision when their eyes are aligned, and most of them show no reduction in stereoacuity when compared with age-matched controls.

\section{Discussion}

Our results agree with previous reports that, in contrast to patients with constant strabismus, patients with $\mathrm{X}(\mathrm{T})$ generally retain their stereo $3 \mathrm{D}$ vision. They are generally reported to have good stereoacuity at short viewing distances but to have more variable distance stereoacuity, probably because $\mathrm{X}(\mathrm{T})$ patients have worse control of fixation at long viewing distances. ${ }^{20-24}$ Previous studies of stereo vision in patients with $\mathrm{X}(\mathrm{T})$ have used clinical stereo tests such as the Titmus, Frisby, and Randot tests. These enable the experimenter to present only a predefined, limited set of disparities at any given viewing distance. Thus, the measured stereoacuity is quantized, potentially obscuring subtle differences between groups. Typically, only small numbers of tests are available at a given disparity, so a single mistake or lucky guess can result in a large shift in measured stereoacuity. In addition, some of these tests present monocular cues, such as monocularly visible shifts in contours (Titmus test) or monocular motion parallax (Frisby test), which observers could in theory exploit to perform the task even in the absence of stereo vision.

In this study, we measured stereoacuity in $\mathrm{X}(\mathrm{T})$ patients at a viewing distance of $120 \mathrm{~cm}$ using random-dot stereo stimuli, which present no monocular cues. Images were generated dynamically according to a Bayesian staircase, which meant that the stimulus disparities presented could be set to those most informative for obtaining the stereoacuity of that participant and the same stimulus was never presented twice because the random dots were scattered randomly on each trial. Consistent with previous studies, ${ }^{20,25}$ a large majority of our patients had normal stereoacuity. Interestingly, the two X(T) patients who were most impaired compared with controls were the two oldest (X04 [16 years] and X05 [22 years]). These two patients also had some of the largest interocular differences in acuity ( 0.3 and $0.1 \log$ MAR, respectively; Table 1$)$, but interocular acuity difference does not seem to preclude good stereoacuity measures because a younger patient, X06, who had an even larger interocular acuity difference of 0.475 , performed normally. This raises the possibility that stereo vision may be initially preserved in $\mathrm{X}(\mathrm{T})$, but if the condition persists into the teenage years, especially with an interocular acuity difference, stereo vision may ultimately begin to be damaged. Further study would be required to confirm this speculation.

\section{EXPeriment 2: One versus Two Faces}

Having established that our patients showed near-normal stereo vision during binocular alignment, we then examined diplopia under the same conditions. In this experiment, participants viewed toy faces such as the examples in Figure $2 \mathrm{~A}$, presented dichoptically, one in each eye. They were asked to report the number of faces they perceived.

\section{Specific Methods}

Between trials, participants viewed a dichoptic butterfly and net on a binocular, zero-disparity forest background (Fig. 2B). Participants were asked to tell us "if the butterfly flies out of the net." This would indicate that the eyes had become misaligned.

During trials, participants viewed toy faces approximately $6^{\circ} \times 8^{\circ}$ in extent. These were presented for $400 \mathrm{~ms}$, which has been reported to be the optimal stimulus duration to elicit suppression. ${ }^{26}$ Identical face images were presented in the center of the screen for one eye and on the left or right side of the screen for the other eye (Fig. 3). The task was to indicate whether one or two faces were perceived. A third button offered a "don't know" option. We used the method of constant stimuli, with at least 10 presentations per position and eye

Figures $3 \mathrm{~A}$ and $3 \mathrm{~B}$ sketch the two sorts of test trials used in this experiment. The images were presented either to the fovea of the right
A
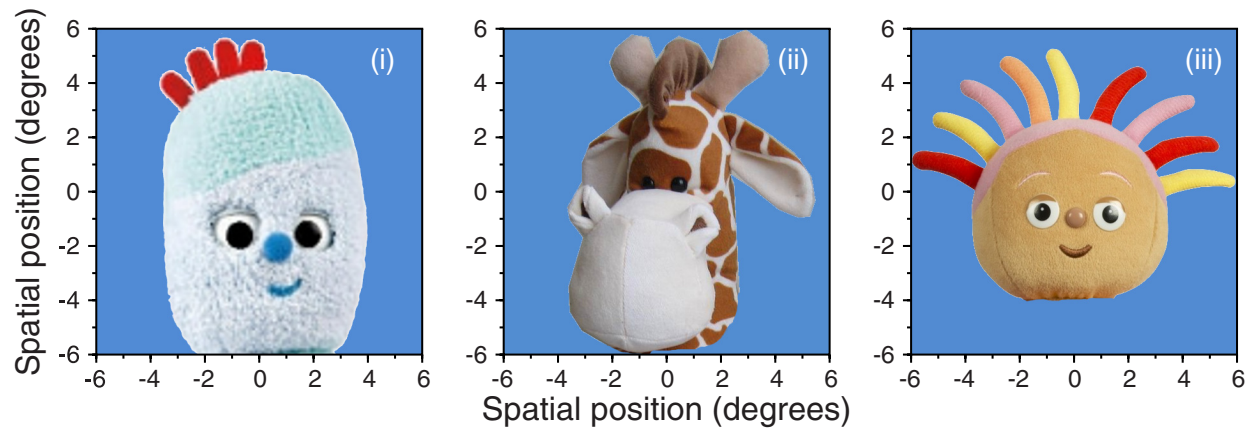

B

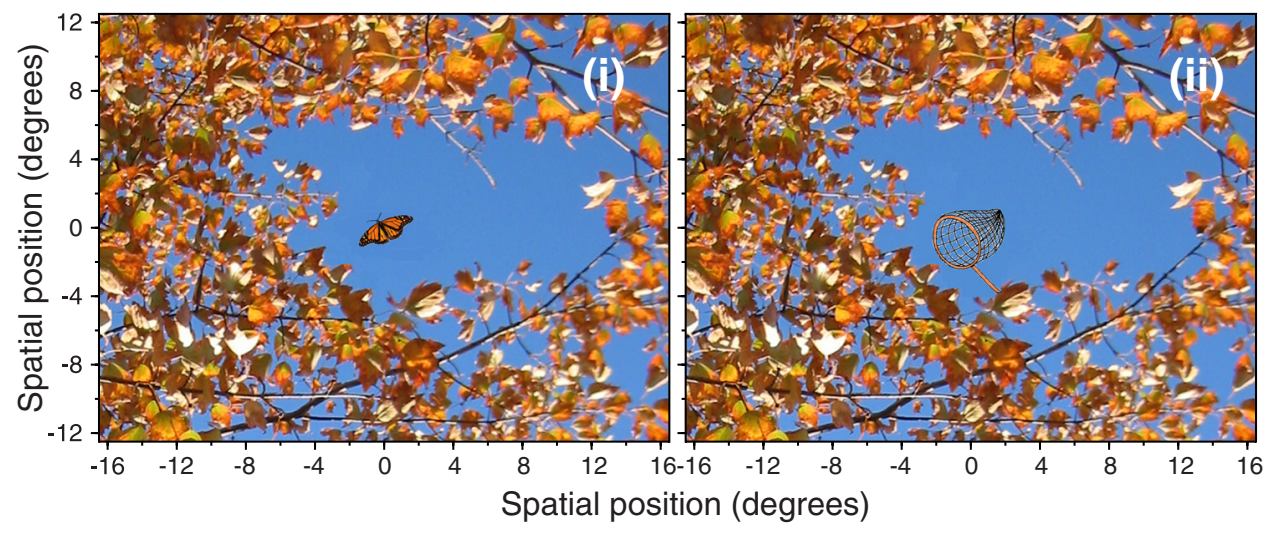

FigURE 2. Examples of stimuli used in the experiments. (A) Examples of toy faces used in the suppression experiments. (B) Nonius image to ensure fixation and to check deviation. The foliage background has zero-disparity, and the butterfly and the net at center were presented dichoptically to the left eye (Bi) and the right eye (Bii). 
A

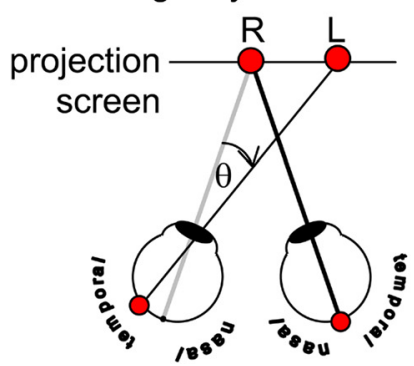

B

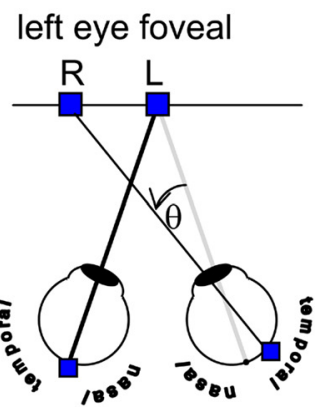

C

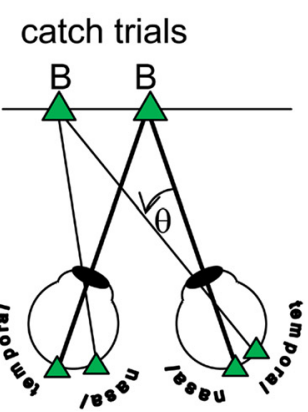

FigurE 3. Sketch of the three conditions in experiment 2 , showing the meaning of the symbols used in Figures 4 and 6. (A) Right eye foveal condition (red circles): Identical face images are presented to the fovea of the right eye and at varying positions $\theta$ along the horizontal retinal meridian of the left eye. (B) Left eye foveal condition (blue squares): Identical images are presented to the fovea of the left eye and at varying positions along the horizontal retinal meridian of the right eye. In each case, nega-

tive values of $\theta$ mean that the nonfoveal image is presented to the nasal hemiretina and positive values to the temporal hemiretina. (C) Catch trials (green triangles): Identical images are presented to the foveas of both eyes and at the same position on the horizontal meridian of both eyes. The labels $L, R$, and $B$ indicate whether an image on the projection screen was visible to the left or the right eye, or to both. The task was to indicate how many faces (one or two) were seen. Thick lines: optic axes of the two eyes, shown in black when the fovea is viewing an object and gray when the fovea is not stimulated. Thin black lines: projection of nonfoveal images onto the retina. These conventions will be maintained in subsequent figures.

eye and at different peripheral positions along the horizontal axis of the left eye (Fig. 3A; results shown with red circles in Fig. 4) or to the fovea of the left eye and at different peripheral positions in the right eye (Fig. 3B; results shown with blue squares). Thus, one eye's image was always foveal and the other varied. The angle $\theta$ is the retinal position of the nonfoveal image and thus also the horizontal disparity between the two eyes' images. We also included catch trials (Fig. 3C; results shown with green triangles in Fig. 4), in which both a foveal and a peripheral image were presented to both eyes. Here, assuming the eyes are aligned, the only possible correct answer was " 2 faces." These trials enabled us to test that participants understood the task and were motivated and able to report their percept correctly, an important control for our child participants. The fact that participants almost always reported two faces on these catch trials and never reported seeing more than two faces indicated that the participants were performing the task as required and maintaining alignment.

To summarize our data, we fitted cumulative Gaussian functions independently to nasal and temporal positions. The fitted function was $\mathrm{P}(\theta)=0.5 \times \mathrm{BPE}+(1-\mathrm{BPE}) \times 0.5\left(1+\operatorname{erf}\left[\left(\theta-\mu_{\mathrm{N}}\right) /\left(\sigma_{\mathrm{N}} V 2\right)\right]\right)$ if $\theta<$ 0 , and $\mathrm{P}(\theta)=0.5 \times \mathrm{BPE}+(1-\mathrm{BPE}) \times 0.5\left(1-\operatorname{erf}\left[\left(\theta-\mu_{\mathrm{T}}\right) /\left(\sigma_{\mathrm{T}} \sqrt{ } 2\right)\right]\right)$ if $\theta \geq 0$, where BPE represents the probability that the participant made a random button press error; this was fixed at 0.01 . The parameters $\mu_{\mathrm{N}}, \sigma_{\mathrm{N}}, \mu_{\mathrm{T}}$, and $\sigma_{\mathrm{T}}$ were fitted to the data by the method of maximum likelihood assuming simple binomial statistics. Data from both eye conditions (left/right eye foveal) were always similar and so were fitted together. Confidence intervals on the fitted parameters were estimated by bootstrap resampling. In other words, for each disparity, we generated random deviates from a simple binomial model using the same number of trials as performed at that disparity and taking the fitted function as our estimate of the binomial probability. These simulated data were then fitted with a new cumulative Gaussian function exactly as described, resulting in a different estimate of the four fit parameters. This process was repeated 1000 times, and the $95 \%$ confidence interval on each fitted parameter was estimated as the range from the $2.5 \%$ percentile to the $97.5 \%$ percentile of these 1000 values.

\section{Results for Controls}

Figure 4 shows the results obtained for one control participant. The remaining controls gave similar results (data not shown). The symbols (blue squares, red circles, and green triangles) represent the probability of seeing one face, as a function of the horizontal disparity $\theta$ (which in our experiment is given by the eccentricity of the peripheral face). The proportion of "don't know" responses was smaller than for Experiment 1, at $2 \%$ averaged across participants. This reflected the use of method of constant stimuli rather than a Bayesian staircase. As Figure 4 shows, most data were collected in regions in which the percept was unambiguously of either one face or two faces, and only a small fraction of trials used disparities in which the response was near chance.

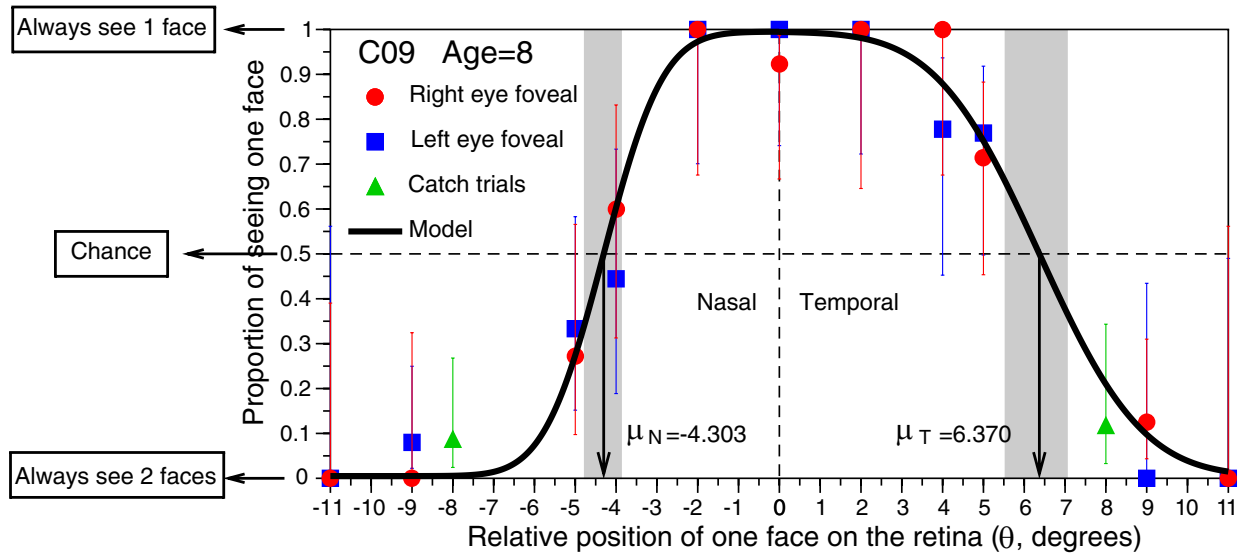

Figure 4. Results for one control participant. Right eye foveal condition (red dots, Fig. 3A): Identical face images are presented to the fovea of the right eye and at varying positions $\theta$ along the horizontal retinal meridian of the left eye. Left eye foveal condition (blue squares, Fig. 3B): Identical images are presented to the fovea of the left eye and at varying positions along the horizontal retinal meridian of the right eye. In each case, negative values of $\theta$ mean that the nonfoveal image is presented to the nasal hemiretina and positive values to the temporal hemiretina. Catch trials (green triangles, Fig. 3C). Identical images are presented to the foveas of both eyes and at the same position on the horizontal meridian of both eyes. Symbols represent the proportion of trials on which one face was reported as a function of the relative position of one face along the horizontal axis. Error bars: $95 \%$ confidence limits assuming binomial variability; the limits were obtained using the score confidence interval. ${ }^{17}$ Black curve: function fitted as described in Specific Methods for Experiment 2. Arrows: $50 \%$ thresholds $\mu_{\mathrm{T}}$ and $\mu_{\mathrm{N}}$. Shaded area: $95 \%$ confidence interval estimated for the threshold. 
The results were exactly as expected given the known properties of binocular vision. When horizontal disparities were small, human observers fused the two eyes' images into a single percept. When disparities exceeded Panum's fusional limit, they experienced diplopia, seeing both eyes' images separately. Consistent with this, in experiment 2 when both faces were on the fovea $\left(\theta=0^{\circ}\right)$ or very close to the fovea, participants reported seeing only one face, consistent with fusion. Sometimes participants spontaneously reported seeing the face closer or more distant, depending on the sign of binocular disparity. As we moved from the fovea to the nasal or temporal hemiretina $\left(|\theta|=4^{\circ}-7^{\circ}\right)$, the proportion of trials reporting two faces increased. For presentations far from the fovea $\left(|\theta|>7^{\circ}\right)$, two images were always perceived (the proportion of "one face" judgments was equal to 0 ), indicating diplopia. This consistent pattern of results was found in all 12 control participants. Additionally, all participants almost always gave the correct response on the catch trials (green triangles; perfect performance would place these triangles on the horizontal axis). This indicated that our participants were reliably reporting their percepts.

To quantify the shift from fusion to diplopia, we fitted a descriptive function to our data, shown by the black curve in Figure 4 (see Specific Methods for details). This enabled us to estimate $\mu$, the difference in image position at which the observer is equally likely to report seeing one or two faces. $\mu$ is a measure of Panum's fusional limit. It is marked with downward arrows in Figure 4.

In Figure 4, the value of the fusional limit $\mu$ depends on whether the nonfoveal image is moved into the temporal or nasal retina. This was true for most of our controls. We therefore separately fitted $\mu_{\mathrm{N}}$ for the nasal case (uncrossed or far disparities) and $\mu_{\mathrm{T}}$ for temporal (crossed or near disparities). Figure $5 \mathrm{~A}$ shows a scatterplot of the $50 \%$ fusional limit for the nasal case, $\mu_{\mathrm{N}}$, plotted against that for the temporal case, $\mu_{\mathrm{T}}$, for our 12 controls. In every case where there is a significant difference, $\mu_{\mathrm{N}}<\mu_{\mathrm{T}}\left(\mu_{\mathrm{N}}=3.9^{\circ} \pm 1.7^{\circ} ; \mu_{\mathrm{T}}=5.1^{\circ} \pm 1.6^{\circ}\right.$; mean \pm SD for 12 controls). This difference is highly significant $(P=0.004$, paired $t$-test $)$. This asymmetric pattern of results means that the transition from fusion to diplopia occurs at smaller disparities when the nonfoveal image is presented in the nasal retina than when it is presented in the temporal retina. Results were not affected by which eye viewed the eccentric image; therefore, the asymmetry is not explained by whether the mean visual direction was left or right.

Figure 5B shows nasal and temporal fusional limits plotted as a function of age. Both decline with age (correlation coeffi- cient between $\mu$ and age is -0.34 for $\mu_{\mathrm{N}}$ and -0.64 for $\mu_{\mathrm{T}}$; only the latter was significant; $P=0.03$ ). This may represent a genuine decrease in Panum's fusional area with age or a change in criterion. It is possible that small children tended to report diplopic images as "one face" if they substantially overlapped, whereas older participants reported these as "two." The point of interest is the comparison with $\mathrm{X}(\mathrm{T})$ patients, to which we now turn.

\section{Results for X(T) Patients}

Figure 6 shows the results for $12 \mathrm{X}(\mathrm{T})$ patients. In contrast to controls, almost all patients show a marked nasal/temporal asymmetry. For small binocular disparities $\theta$, patients report seeing one face, just as controls do. As the peripheral image moves further into nasal hemiretina ( $\theta$ becomes more negative), patients report seeing two faces - that is, they experience diplopia just as controls do. As for controls, we used our fitted function to estimate the image separation at which $\mathrm{X}(\mathrm{T})$ patients were equally likely to report seeing one or two faces, $\mu_{\mathrm{N}}$. As for controls, we found that these fusional limits decreased with age (Fig. 5C). However, we also found that the fusional limits were consistently larger for $\mathrm{X}(\mathrm{T})$ patients than for age-matched controls $\left(\mu_{\mathrm{N}}=3.9^{\circ} \pm 1.7^{\circ}\right.$ for controls (mean $\pm \mathrm{SD}$, black circles in Fig. $5 \mathrm{C}$ ), $6.2^{\circ} \pm 2.3^{\circ}$ for patients (blue squares); $P=0.0129, t$-test) Thus, when the peripheral image moved into nasal hemiretina, $X(T)$ patients shifted from fusion to diplopia as did controls, but they can apparently fuse larger disparities than controls.

When the peripheral image moved into the temporal hemiretina, a big difference emerged between $\mathrm{X}(\mathrm{T})$ patients and controls. Most patients reported perceiving only one face even at disparities of many degrees, in contrast to controls who experienced diplopia. This result was very clear in 9 of 12 patients. Of the remaining three, patient X08, aged 6, was distracted during the experiment, as indicated by the fact that she performed at chance during the catch trials. Nevertheless, her results do suggest the same nasal/temporal asymmetry as in the other patients. Two of 12 patients, X10 and X11, performed more like controls. Despite some asymmetry, they did clearly experience diplopia at sufficiently large disparities, even when the peripheral image was presented to the temporal hemiretina.

There was no evidence of any difference in the propensity of patient participants to choose the "don't know" response. On average across the 12 patients, patients responded "don't know" on $2 \%$ of trials, the same proportion as controls.
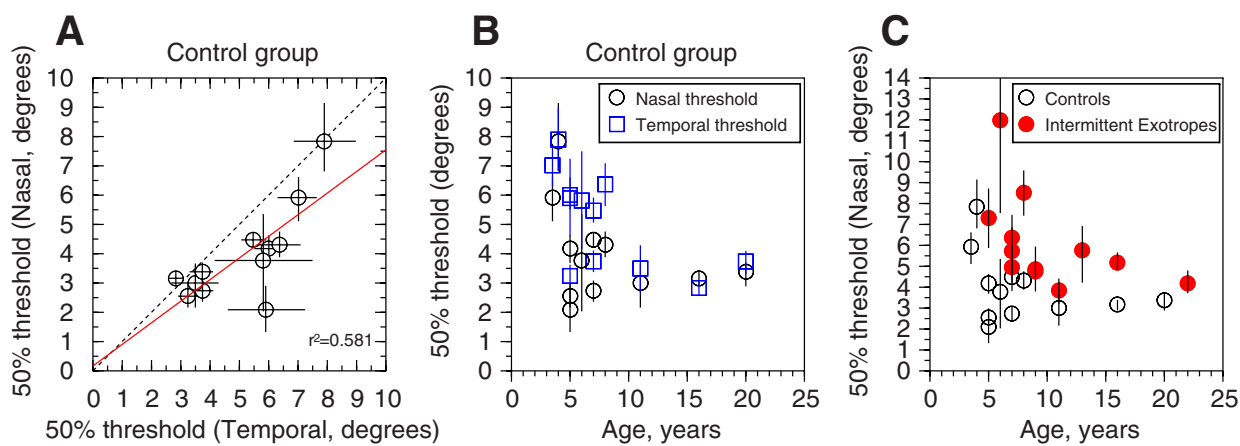

FIGURE 5. Panum's fusional limits obtained from experiment 2 (see Fig. 4). (A) Nasal 50\% thresholds $\left(\mu_{\mathrm{N}}\right)$ as a function of temporal $50 \%$ thresholds $\left(\mu_{\mathrm{T}}\right)$. Dotted line: linear relationship. Red line: regression line $\mu_{\mathrm{N}}=0.738 \times \mu_{\mathrm{T}}+0.167$. The coefficient of determination was $r^{2}=0.581$. (B) $50 \%$ thresholds as a function of age. Open circles: nasal thresholds; blue squares: temporal thresholds. (C) Nasal thresholds as a function of age. Open circles: control participants; red circles: patients with intermittent exotropia. In all panels, error bars show the $95 \%$ confidence intervals obtained by running 1000 Monte Carlo simulations. 


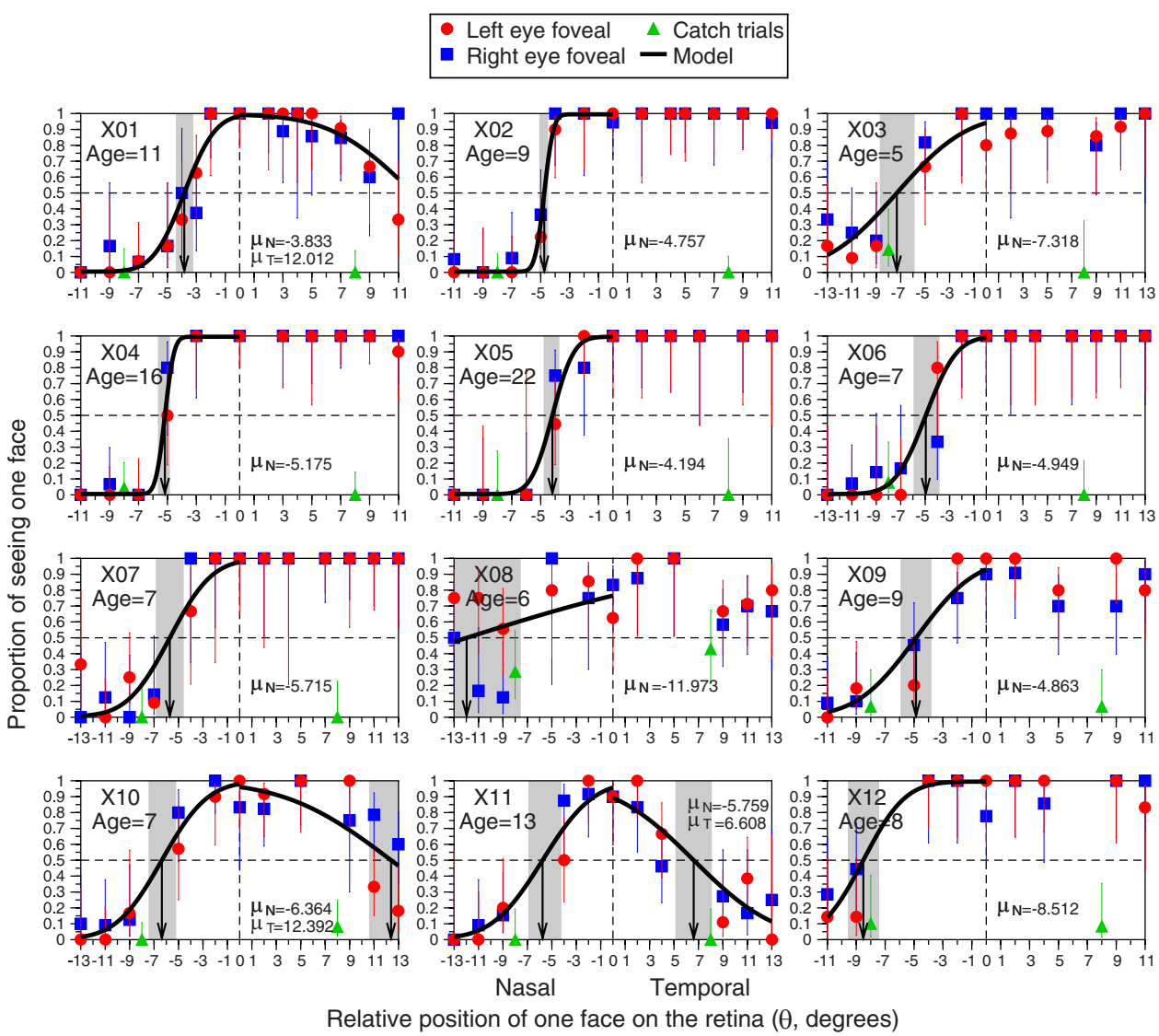

Figure 6. Results from experiment 2 for $12 \mathrm{X}(\mathrm{T})$ patients, in the same format as Figure 4.

\section{Discussion}

$\mathrm{X}(\mathrm{T})$ patients usually do not report experiencing diplopia during their periods of exodeviation, presumably because they have developed specific neuronal mechanisms to avoid diplopia. Previous studies have almost all examined patients during periods of exodeviation, where there are both retinal and nonretinal cues to the misalignment. In contrast, our results were obtained during correct binocular alignment, proving that antidiplopic mechanisms in $\mathrm{X}(\mathrm{T})$ can be triggered by purely retinal information and do not require an oculomotor signal indicating that deviation has occurred. In experiment 2 , the retinal trigger consisted of identical images in the fovea of one eye and in the temporal hemiretina of the other eye. The trigger occurred independently of which eye saw the foveal image and which saw the temporal image. However, when the peripheral image was presented in the nasal hemiretina, antidiplopic mechanisms were not triggered and $\mathrm{X}(\mathrm{T})$ patients reported diplopia, just as controls did. Although the patients behaved qualitatively like controls in this situation, there was a small quantitative difference: our $\mathrm{X}(\mathrm{T})$ patients could fuse larger disparities than controls with no eye movement disorder. ${ }^{7,27}$ We speculate that this might have occurred because the poorer binocular control of $\mathrm{X}(\mathrm{T})$ patients means they experience a wider range of retinal disparities in their day-today life than controls do.

It seems likely that this nasal/temporal asymmetry stems from the geometry of exodeviation. If a patient attempts to fixate an object while exodeviating, the image will fall at the fovea of the fixating eye but in the temporal hemiretina of the deviating eye. Thus, as Figure 7 shows, patients could experience retinal images such as the positive $\theta$ stimuli in experiment1 (peripheral image in temporal hemiretina) during episodes of exodeviation in their daily life. Conversely, retinal images such as the negative $\theta$ stimuli of experiment 2 (peripheral image in nasal hemiretina) would only occur outside the laboratory if one eye deviated inward. Our patients experience ocular deviation outward during their daily lives but do not experience deviation inward. Hence, as Figure 6 shows, they have developed means of preventing diplopia for positive $\theta$ but not for negative $\theta$.

Future investigations of $\mathrm{X}(\mathrm{T})$ will be greatly simplified by our demonstration that antidiplopic mechanisms can be triggered during orthotropia by purely retinal information. This obviates the need to patch the eye for 60 minutes before an experiment to induce exodeviation. ${ }^{8}$ We have demonstrated that, in $\mathrm{X}(\mathrm{T})$, a stimulus in one eye can trigger suppression of an identical image presented many degrees away in the other eye. This process should be particularly amenable to study by functional magnetic resonance imaging. Because the two eyes' images are at different retinotopic locations, the activation caused by each can be studied without "cross-contamination" from other. Potentially, such studies could identify the cerebral area in which suppression occurs, giving important new insights into how and where visual stimulation results in conscious perception.

\section{Experiment 3: Location of the Percept}

In experiment 2, we have shown that most patients with $\mathrm{X}(\mathrm{T})$ do not experience diplopia if the same image is presented to the fovea of one eye and to the temporal hemiretina of the other eye, even while their eyes are aligned correctly. This is consistent with previous reports of suppression scotomas in the temporal hemiretina during ocular deviation in intermittent and constant exotropia. ${ }^{5,6,28-30}$ In these reports, the suppression scotoma was, of course, in the nonfixating eye. Given that 


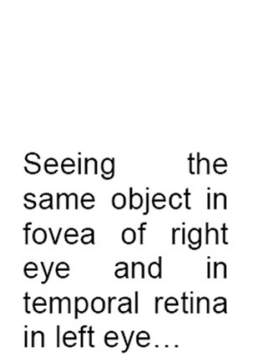

Figure 7. Diagrams show how the retinal images produced by our dichoptic experimental stimuli during binocular alignment could also be produced by real objects encountered outside the laboratory if viewed with exodeviation. (A, B) Dichoptic images are presented on a projection screen; $3 \mathrm{D}$ glasses ensure that each image is seen by only one eye, as indicated by the labels $L$ and $R$ and the black lines showing the projection into each eye. (C, D) Images are produced by a real object projecting to both eyes, as indicated by the label $B$. If one eye is exodeviating, this can result in the same retinal images as in (A) and (B).

...could mean the object is being fixated by the right eye and the left eye is exodeviating.
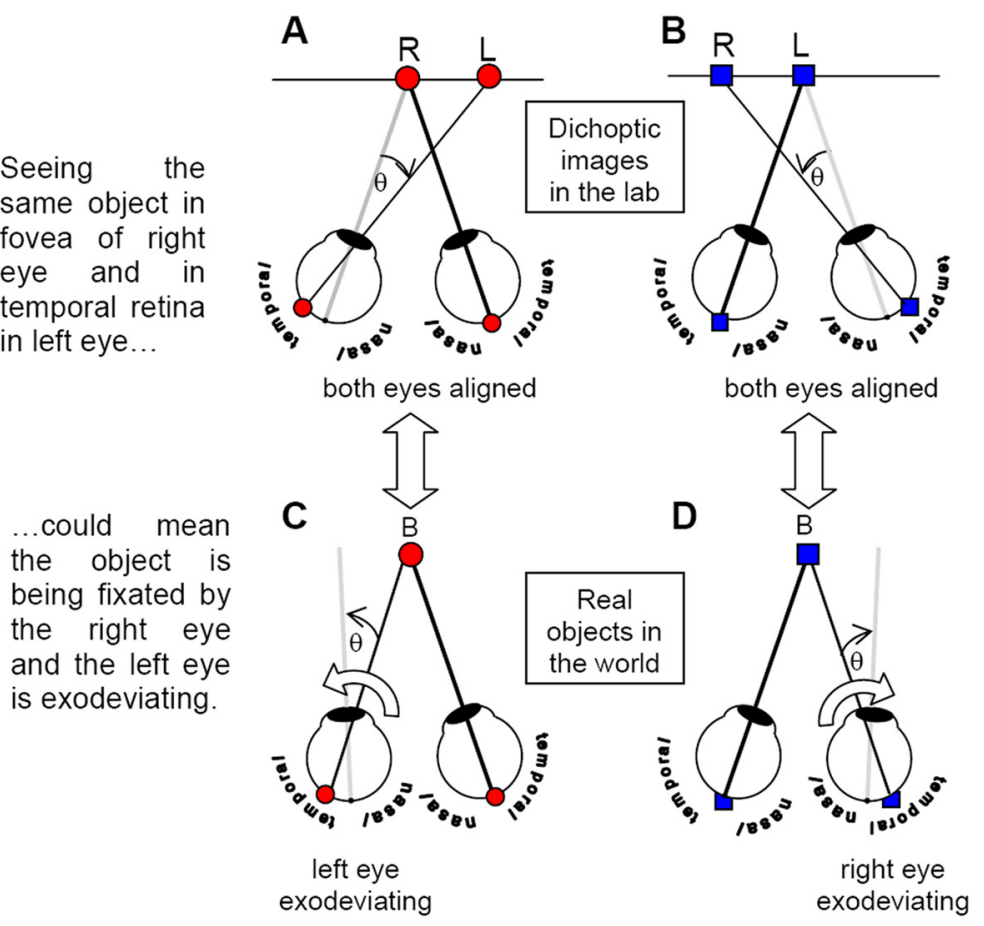

$\sqrt{B}$
Seeing the same object in fovea of left eye and in temporal retina in right eye... our patients were correctly fixating with both eyes during experiment 2, it was not obvious which eye was suppressed.

To probe this, we performed experiment 3 on 8 of our 12 $\mathrm{X}(\mathrm{T})$ patients. We were not able to recall participants $\mathrm{X} 01$ and X04 for this experiment, and we did not perform it in X08 because this 6-year-old participant had become distracted during experiment 2 , as indicated by chance performance on catch trials. In patient X11, we did not find any antidiplopic mechanisms to examine.

\section{Specific Methods}

For each $\mathrm{X}(\mathrm{T})$ patient who participated in experiment 3, we selected a retinal location that reliably triggered the antidiplopic mechanisms. To achieve this, we chose a disparity $\theta_{\text {test }}$ large enough that the patient reliably reported seeing two faces when the peripheral image was presented in the nasal hemiretina but reported seeing only one face when the peripheral image was in the temporal hemiretina (Fig. 8). The smallest value of $\theta_{\text {test }}$ used was $8^{\circ}$ (maximum $11^{\circ}$ ). This is well outside Panum's fusional limit, as demonstrated by the fact that $\mathrm{X}(\mathrm{T})$ patients reliably perceived two faces when the peripheral image was $8^{\circ}$ nasal, and control participants reliably perceived two faces when the peripheral image was $8^{\circ}$ either nasal or temporal. Thus, the fact that patients perceived only one face when the peripheral image was at $\theta_{\text {test }}$ temporal cannot indicate normal binocular fusion but rather must indicate some mechanism specific to $\mathrm{X}(\mathrm{T})$.

Having selected an appropriate eccentricity $\theta$, we then conducted experiment 3 presenting the peripheral image in the temporal hemiretina of either the left or the right eye (Fig. 8C). Based on experiment 2 , we already knew that only one image would be perceived in this position. Accordingly, each participant's task was now not to report the number of perceived faces but to report the location of the single perceived face. The three response buttons were used to report seeing it on the left-hand side of the screen, in the center, or on the right.

Figure 9 shows the pattern of responses we would expect for experiment 3 in different situations. Figure 9A shows the pattern expected from an uncooperative or unmotivated participant who is simply pressing buttons at random. Note that these responses can be distinguished from those of the participant who reliably reports his or her percepts, but where there is no rule governing which of the dichoptic images is perceived. The brain suppresses either one of the two images completely at random on every trial. That would produce the pattern shown in Figure 9B.

The remaining rows show what happens under various deterministic rules governing which eye or image is suppressed. Figure 9C shows the pattern expected if the patient always perceives the foveal image and suppresses the temporal image, irrespective of in which eye it falls. This strategy makes most sense if the patient habitually fixates with either eye, so that the brain has to use the available retinal information to "decide" which eye to suppress. A sensible strategy would be to assume that the eye receiving the foveal image is fixating, whereas the eye receiving the same image in the temporal retina is exodeviating and should be suppressed. We would then expect the patient always to report seeing the image in the middle of the screen, where he or she is fixating.

Figures 9D and 9E show the pattern expected if the patient consistently suppresses the same eye whenever the retinal images indicate exodeviation. For example, Figure 9D shows the predicted pattern if the patient always suppresses the right eye and perceives whatever image is presented to the left eye. The response will be "right" when the left eye's image is temporal (red circles) and "center" when the left eye's image is foveal (blue squares). Conversely, if the left eye is always suppressed, the pattern of responses will be as shown in Figure 9E. These two strategies make most sense if the exodeviation always affects the same eye.

\section{Results}

Results are shown in Figure 10. Experiment 3 contains only two stimuli, sketched at the bottom of the figure (Fig. 10I; red circles indicate right eye foveal, with center image presented in the fovea of right eye and right image presented in the temporal retina of left eye; blue squares indicate left eye foveal, with center image presented in the fovea of left eye and left image presented in the temporal retina of right eye). Each stimulus is presented several times. On every trial, participants are asked where on the screen the image is perceived. There are three possible responses-left, center, and right-labeled above each stimulus in Figure 10. The height of the bars for each patient indicates how their responses for each stimulus 


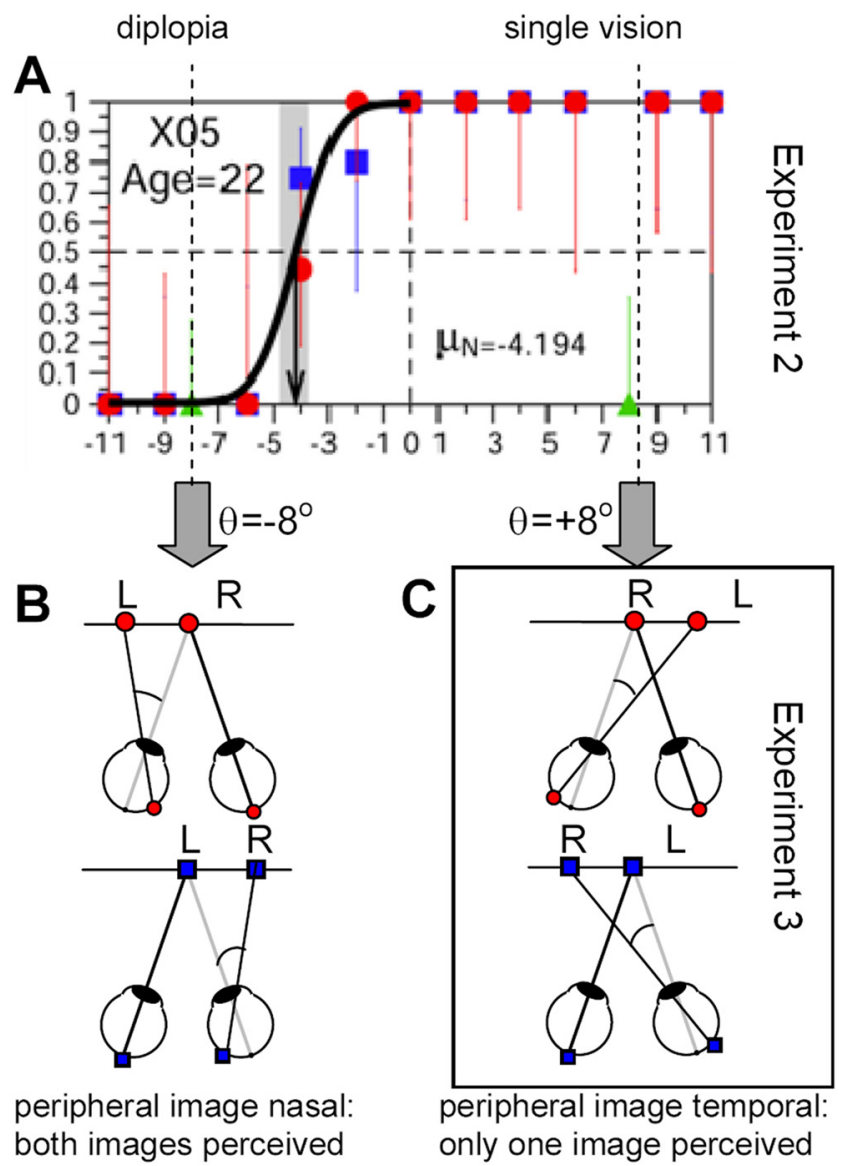

Figure 8. How the results of experiment 2 (A) are used to select a suitable value of $\theta$ for experiment 3 . We seek a value of $\theta$, which results in diplopia when the peripheral image is nasal ( $\theta$ negative, $\mathbf{B}$ ) and in single vision when the peripheral image is temporal ( $\theta$ positive, C). For X05, a suitable value is $\theta=8^{\circ}$. Experiment 3 then uses two stimuli with $\theta=8^{\circ}(\mathrm{C})$, one in which images are presented to the fovea of the right eye and $8^{\circ}$ temporally in the left eye (red circles) and one in which images are presented to the fovea of the left eye and $8^{\circ}$ temporally in the right eye (blue squares)

were divided between the three possibilities, shown with open bars. Perfect consistency corresponds to one of the three bars being filled up to 1 and the others being empty.

In fact, many patients showed some variability, giving different answers on successive presentations of the same stimulus, despite that when asked they reported consistently seeing only one face. However, even with this variation, no patients showed the pattern of Figure 9A. This gives us confidence that all patients, despite the young ages of some of them, were correctly reporting their perceptions and not responding randomly, suggesting that the same stimulus can elicit different percepts. Furthermore, there was a lot of intersubject variation even between patients with perfectly consistent responses. For example, patient X05 always responded "center" when the right eye's image was foveal and the left was temporal, and patient X02 always responded "right" for the same stimulus.

Figure 10 groups patients according to the pattern of their results. All our patients habitually deviated one eye in particular, as indicated below each patient's identifying code in Figure 10. Both which eye deviated and which had lower acuity seemed to affect the pattern of results. Patients X05 and X06 had the largest difference in visual acuity of any of the eight patients who performed experiment 3 (difference in logMAR scores 0.1 and 0.475 , respectively), and both consistently sup- pressed the eye with lower acuity, which in each case was also the eye that habitually exodeviated. Patients X03, X07, and X09 had rather similar visual acuity in both eyes (difference in logMAR scores $0.075,0.000$, and 0.025 ), and all tended to suppress the temporal image, irrespective of in which eye it fell (Figs. 10C-E).

The remaining two patients, X10 and X12, habitually deviated their left eye. They both reliably reported seeing the face on the left if the right eye's image was temporal, consistent with suppressing the left eye's image (Figs. 10A, 10B). However, if the left eye's image was temporal and the right eye's was foveal, they did not report seeing the face at the center of the screen. Instead, they always (X12) or primarily (X10) reported seeing the face to the right of the screen.

In patients $\mathrm{X} 02$ and $\mathrm{X} 07$, we were able to carry out two more control experiments, described in the Appendix. Control experiment 1 confirmed that these patients did not have a permanent scotoma in the temporal retina. The suppression scotoma demonstrated in experiments 2 and 3 was temporary and was triggered by the fact that the retinal images simulated exodeviation. In control experiment 2 , we confirmed that the suppression occurred only if the retinal images simulated exodeviation. Initially, the dichoptic images were identical apart from an offset. When the dichoptic images were different, these $\mathrm{X}(\mathrm{T})$ patients showed diplopia similarly to controls. Similar results have already been reported with constant strabismus. ${ }^{28,30-32}$

\section{Discussion}

Six of 8 patients showed results consistent with suppression (Figs. 10C-H). Patients X02, X05, and X06 tended to suppress the habitually deviating eye, even if its image was foveal. In 2 of these 3 patients, the habitually deviating eye had much poorer acuity, offering a plausible explanation for why the brain is willing to suppress a foveal image in that eye in favor of a much more eccentric image in the habitually fixating eye. Patients X03, X07, and X09, who had similar acuity in both eyes, were more likely to suppress whichever eye received the temporal image. Interestingly, therefore, although in each of these three patients one eye was defined clinically as the habitually deviating eye, the brain dynamically switched between suppressing left or right eyes according to the instantaneous retinal input.

The results of the remaining two patients, $\mathrm{X} 10$ and $\mathrm{X} 12$, are difficult to reconcile with suppression (Figs. 10A, 10B). For both stimuli, these patients are more likely to report seeing the image on the left or the right of the screen than in the center. If we assume that their single vision was due to suppression, we are forced to conclude that when identical images are presented to the fovea of one eye and to the temporal retina of the other, these patients suppress the foveal image, regardless of which eye views it, and instead perceive the temporal image at $\geq 8^{\circ}$ of eccentricity. This seems highly unlikely.

These patients' results make more sense when interpreted as a form of anomalous retinal correspondence. ${ }^{4,10,33,34}$ In normal retinal correspondence, a point at a temporal eccentricity, $x$, in one eye corresponds to the same visual direction as a point at nasal eccentricity, $-x$, in the other eye. Images falling at corresponding points will be fused if presented simultaneously and perceived as lying in the same visual direction if presented sequentially. Conversely, images presented with large geometric disparities (i.e., the image in the right eye is several degrees away from the point that corresponds geometrically to the left image) produce diplopia. Long-standing oculomotor abnormalities can perturb the normal pattern of retinal correspondence. Patients with $\mathrm{X}(\mathrm{T})$ may show normal retinal correspondence during orthotropia but may shift to anomalous retinal correspondence 


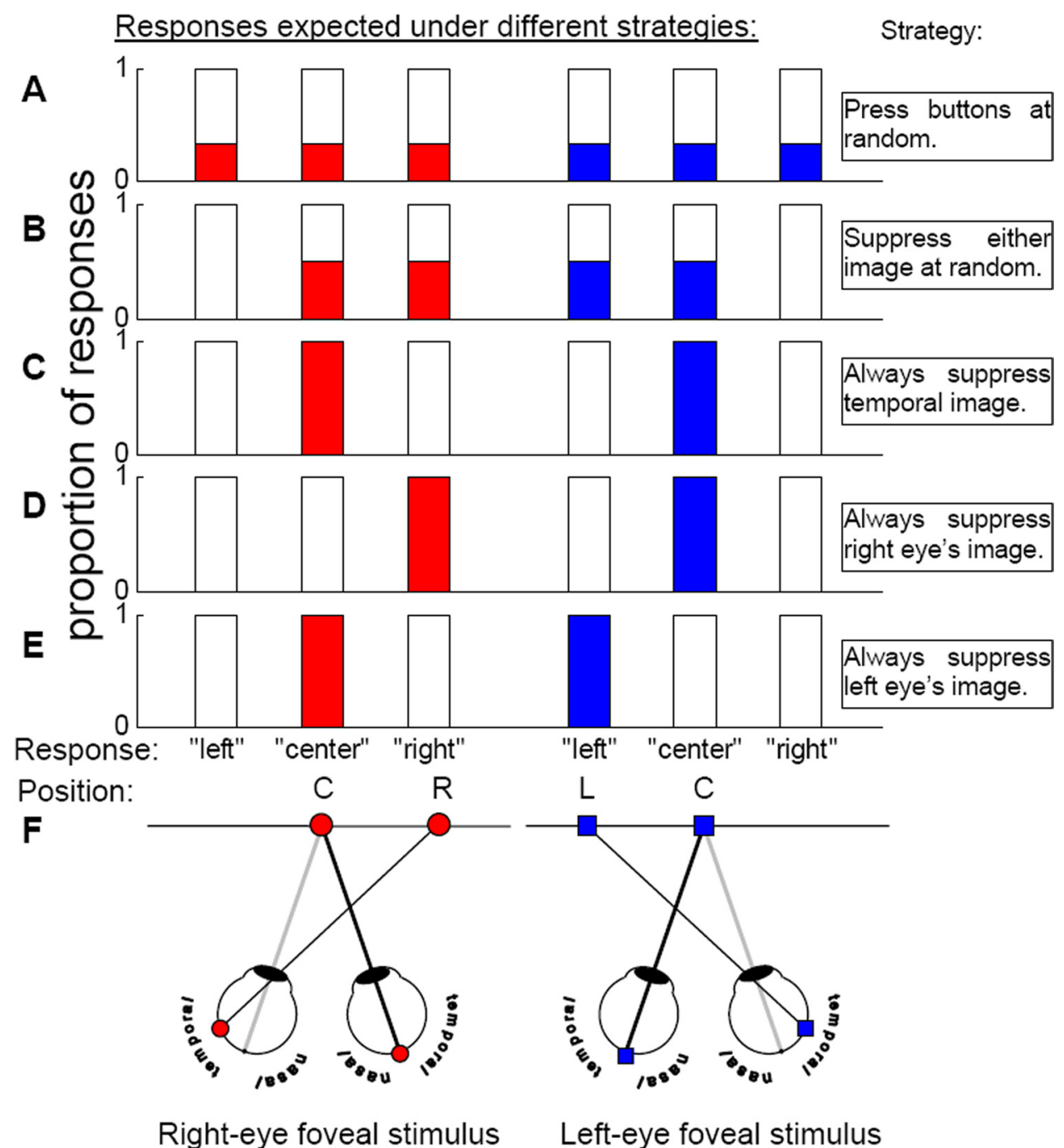

Figure 9. Expected pattern of responses in experiment 3 , under different suppression strategies (B-E) or if the participant does not perform the task but simply presses buttons at random (A). Format as in Figure 10.

Right-eye foveal stimulus Left-eye foveal stimulus

during exodeviation. ${ }^{4,10,11,33,35}$ We know our patients had normal retinal correspondence during orthotropia because they all had functional stereo vision in the normal range of disparities and they all perceived the butterfly as in the net when shown the Nonius image in Figure 2B. However, our experimental images, which simulated exodeviation, might have triggered a shift to anomalous retinal correspondence, possibly through head-centric disparity mechanisms. ${ }^{36,37}$ The lack of diplopia demonstrated in experiment 2 would then reflect not suppression but successful fusion of images with large retinal disparity.

Anomalous retinal correspondence could explain the very consistent results obtained from patient X12 (Fig. 10A), which are difficult to explain by suppression. In normal binocular viewing, an object that projects to the fovea in the right eye and to the temporal retina in the left eye must lie to the right of the midline, whereas an object that projects to the fovea in the left eye and to the temporal retina in the right eye must lie to the left of the midline (for example, compare the solid lines showing the back-projections from the retinal images intersecting in Figs. 3A and 3B). In control participants, the very large disparities in our stimuli $\left(\geq 8^{\circ}\right)$ mean that diplopia occurs, and two faces are perceived. However, if patient X12 could fuse these large retinal disparities and use the mean retinal position to judge visual direction, this would account for her responses in experiment 3 (Fig. 10A). Similarly, anomalous correspondence could also contribute to the results of patients X10, X02, and $\mathrm{X} 09$.

\section{Conclusions}

Experiment 2 showed that, in patients with $\mathrm{X}(\mathrm{T})$, retinal images simulating exodeviation reliably triggered neuronal mechanisms that prevented diplopia, even when the eyes were correctly aligned. This effect was very reliable and highly consistent across patients. Experiment 3 revealed, however, that there is a lot of variation in precisely how these neuronal mechanisms prevent diplopia, both within and between participants. Thus, though most $\mathrm{X}(\mathrm{T})$ patients developed retinatriggered mechanisms to suppress diplopia during their exodeviation, these mechanisms operated in different ways, probably reflecting such factors as which eye deviated, how far, and how often during development.

\section{ApPendix}

\section{Control Experiment 1}

We were able to recall two patients, $\mathrm{X} 02$ and $\mathrm{X} 07$, to conduct two further control experiments. In control experiment 1 , we checked that these patients had no difficulty in perceiving our face images when they were presented eccentrically to each eye individually. In each trial, either an "Igglepiggle" face (Fig. 2Ai) or "giraffe" (Fig. 2Aii) was presented to one eye only. The image was either at the fovea or eccentrically at $4^{\circ}, 8^{\circ}$, or $11^{\circ}$ along the horizontal retinal 


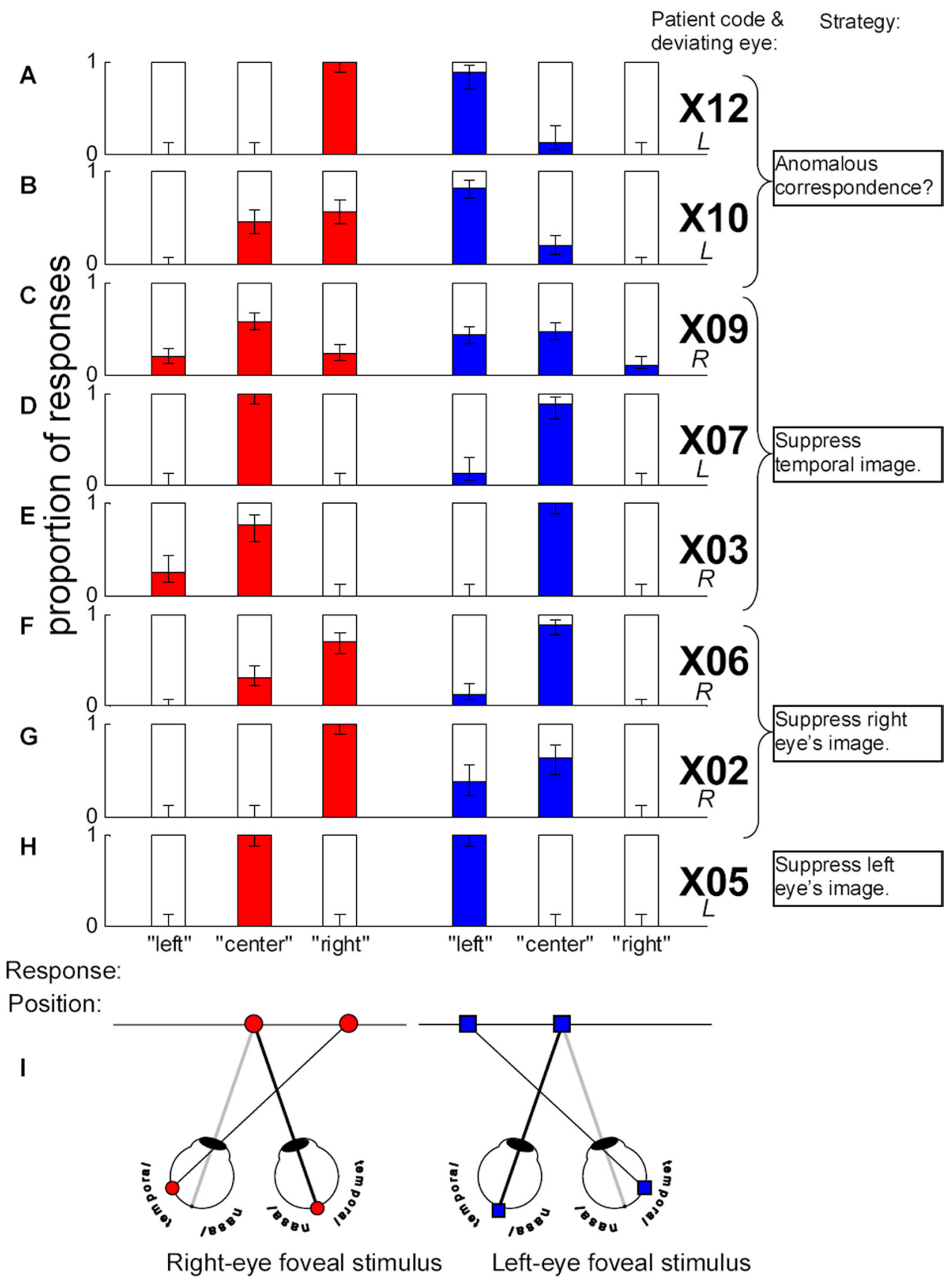

Figure 10. Responses of eight $\mathrm{X}(\mathrm{T})$ patients in experiment 3, grouped according to the pattern of their results. The letter below each patient's identifying code indicates the eye that habitually exodeviates. The diagram at the bottom (I) shows the two stimuli presented in experiment 3; $\mathbf{C}$ is the image presented at the center of the screen. $R$, right; $L$, left. Identical images are presented to the fovea of one eye and to the temporal hemiretina of the other eye. The height of the colored bars in (A-H) shows the proportion of trials in which the participant makes the response indicated on the horizontal axis for each of the two stimuli shown in (I). Error bars show $70 \%$ confidence intervals, assuming simple binomial statistics. meridian in the temporal direction; other details were as for experiment 2 . The task was to identify the character presented. Ten trials were run for each of these four locations in each of the two eyes. Both patients performed this task nearly flawlessly. Patient X07 correctly identified the face on all 40 trials, and X02 made only one error, at the largest eccentricity, but still correctly identified the face on 11 of 12 trials, which is significantly above chance $(P=0.003)$. This demonstrated that these patients were easily able to perceive stimuli in the temporal hemiretina of each eye, if the other eye viewed a blank screen. Suppression was triggered only when the binocular stimulus indicated exodeviation.

\section{Control Experiment 2}

In constant strabismus, suppression is more likely when the images for the left and right eyes are similar. ${ }^{28,30-32}$ To investigate this, in control experiment 2, we repeated ex- periment 2 using three retinal locations $\left(-11^{\circ}, 0^{\circ}\right.$, and $\left.11^{\circ}\right)$ but now using two conditions: identical faces presented to both eyes, as in the original experiment 2 , and different faces presented to the two eyes (for position $0^{\circ}$, the same images were presented to avoid rivalry). Figure 11 shows the results for patients $\mathrm{X} 02$ and $\mathrm{X} 07$ in the same format as Figure 6. Each panel shows the proportion of trials in which patients reported seeing one face, as a function of the relative position of the face on the retina. The top row shows the results when the faces presented in the fovea and in the nasal or temporal retina were identical. This showed the same strong nasal/temporal asymmetry seen in the original experiment 2 several months earlier and demonstrated that the results of experiment 2 are reliable and reproducible across time. The bottom row shows the results when the foveal and peripheral faces were different from one another (e.g., giraffe at the fovea of the left eye, frog in the 

ment 2 for two X(T) patients. Symbols represent the proportion of seeing one face (value of 1) as a function of the relative position of one face along the horizontal axis. Left eye (blue squares): the right eye and other image in different retinal positions along the horizontal axis of the left eye. Negative values correspond to the presentation in the nasal hemiretina, positive values to the temporal hemiretina. Note that in all but one case, the blue squares are partially obscured by red circles. Right eye (red circles): Top row: the same faces are presented to both eyes. Bottom row: different faces are presented in both eyes except for the position at $0^{\circ}$. Control condition (green triangles): In these catch trials, two images were presented in both eyes. Error bars show the 95\% confidence limits assuming binomial variability; the limits were obtained using the score confidence interval.
Figure 11. Results of control experiOne image is presented in the fovea of

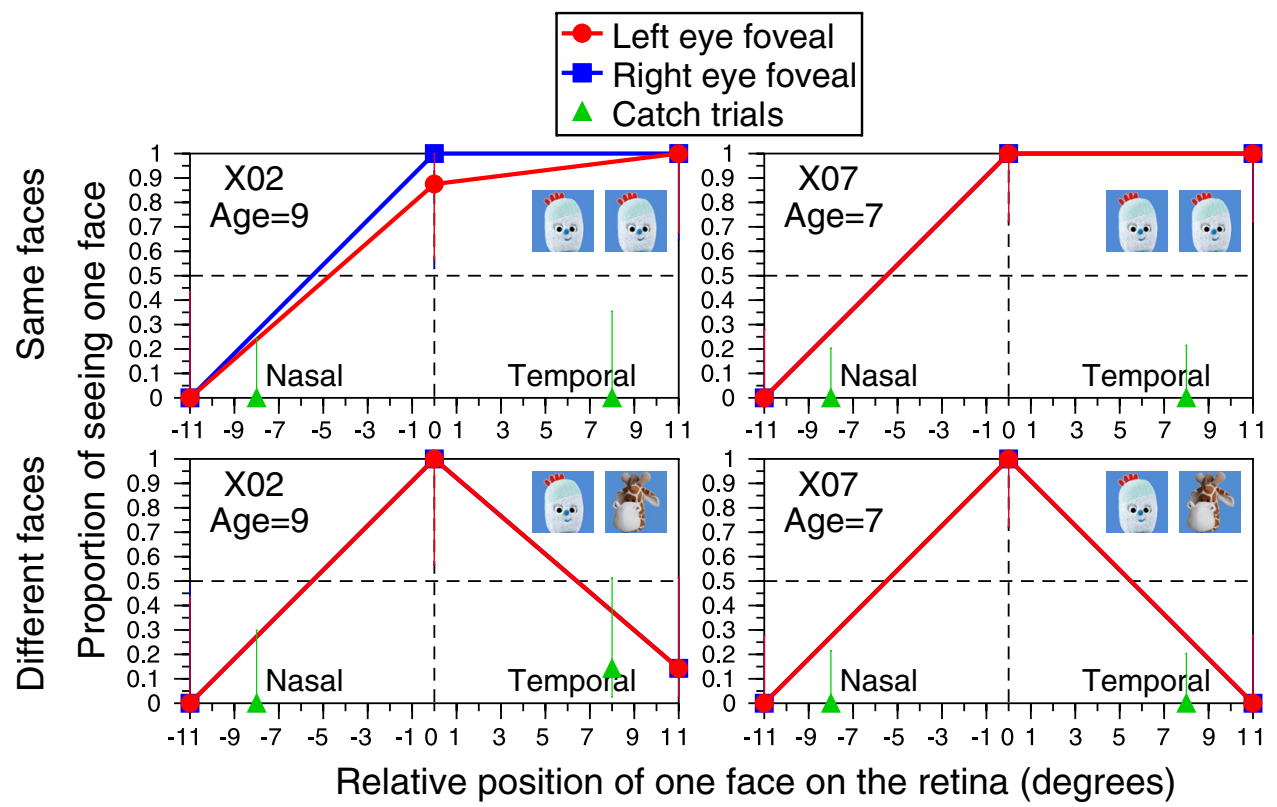

9. Cooper J, Feldman J, Pasner K. Intermittent exotropia: stimulus characteristics affect tests for retinal correspondence and suppression. Binocular Vis Strabismus Q. 2000;15:131-140.

10. Cooper J, Record CD. Suppression and retinal correspondence in intermitent exotropia. Br J Ophthalmol. 1986;70:673-676.

11. Cooper J, Feldman J. Panoramic viewing, visual acuity of the deviating eye and anomalous retinal correspondence in the intermittent exotropia of the divergence excess type. Am J Optom Psychol Opt. 1979;56:422-429.

12. Serrano-Pedraza I, Read JCA. Stereo vision requires an explicit encoding of vertical disparity. $J$ Vis. 2009;9:1-13.

13. Pelli DG. The VideoToolbox software for visual psychophysics: transforming numbers into movies. Spat Vis. 1997;10:437-442.

14. Brainard DH. The psychophysics toolbox. Spat Vis. 1997;10:433436.

ers thank Ignacio Serrano-Blanco for helpful feedback experimental design during the pilot stage, Katharine Read for helpful feedback on experimental design during the pilot stage and for providing the auditory cues, and all the participants and their parents for so generously giving their time to aid vision research.

\section{References}

1. Govindan M, Mohney BG, Diehl NN, Burke JP. Incidence and types of childhood exotropia: a population-based study. Ophthalmology. 2005;112:104-108.

2. Von Noorden G, Campos EC. Binocular Vision and Ocular Motility: Theory and Management of Strabismus. St. Louis: Mosby-Year Book; 2002.

3. Jampolsky A. Differential diagnostic characteristics of intermittent exotropia and true exophoria. Am Orthoptic J. 1954;4:48-55.

4. Cooper J, Medow MD. Major review: intermittent exotropia; basic and divergence excess type. Binocular Vis Eye Muscle Surg $Q$ 1993;8:185-216.

5. Pratt-Johnson JA, Wee HS. Suppression associated with exotropia. Can J Ophthalmol. 1969;4:136-144.

6. Awaya S, Nozaki H, Itoh T, Harada K. Studies of suppression in alternating constant exotropia and intermittent exotropia with reference to effects of fusional background. In: Moore S, Mein J, Stockbridge L, eds. Orthoptics: Past, Present, and Future. New York: Stratton Intercontinental Medical Book Co.; 1976:531546.

7. Pritchard C, Flynn JT. Suppression of physiologic diplopia in intermittent exotropia. Am Orthoptic J. 1981;31:72-79.

8. Melek MD, Shokida MD, Dominguez MD, Zabalo MD. Intermittent exotropia: a study of suppression in the binocular visual field in 21 cases. Binocular Vis Strabismus Q. 1992;7:25-30.
15. Pentland A. Maximum likelihood estimation: the best PEST. Percept Psychophys. 1980;28:377-379.

16. Emerson PL. Observations on maximum-likelihood and Bayesian Psychophys. 1986;39:151-153.

17. García-Pérez MA. Forced-choice staircases with fixed steps sizes: asymptotic and small-sample properties. Vis Res. 1998;38:18611881.

18. King-Smith PE, Grigsby SS, Vingrys AJ, Benes SC, Supowit A. Efficient and unbiased modifications of the QUEST threshold method: theory, simulations, experimental evaluation and practical implementation. Vis Res. 1994;34:885-912.

19. Anderson AJ. Utility of a dynamic termination criterion in the ZEST adaptive threshold method. Vis Res. 2003;43:165-170.

20. Stathacopoulos RA, Rosenbaum AL, Zanoni D, et al. Distance stereoacuity: assessing control in intermittent exotropia. Ophthalmology. 1993;100:495-500.

21. Yildirim C, Altinsoy HI, Yakut E. Distance stereoacuity norms for the mentor B-VAT II-SG video acuity tester in young children and

22. Yildirim C, Mutlu FM, Chen Y, Altinsoy HI. Assessment of central and peripheral fusion and near and distance stereoacuity in intermittent exotropic patients before and after strabismus surgery. Am J Ophthalmol. 1999;128:222-230.

23. Sharma P, Saxena R, Narvekar M, Gadia R, Menon V. Evaluation of distance and near stereoacuity and fusional vergence in intermittent exotropia. Ind J Ophthalmol. 2008;56:121-125.

24. Hatt SR, Haggerty H, Buck D, Adams W, Strong NP, Clarke MP. Distance stereoacuity in intermittent exotropia. Br J Ophthalmol. 2007;91:219-221. methods of forced-choice sequential threshold estimation. Percept young adults. J AAPOS. 1998;2:26-32. 
25. Baker JD, Davies GT. Monofixational Intermittent Exotropia. Arch Opbthalmol. 1979;97:93-95.

26. Joosse MV, Simonsz HJ, Spekreijse H, Mulder PGH, Minderhout HM. The optimal stimulus to elicit suppression in small-angle convergent strabismus. Strabismus. 2000b;8:233-242.

27. Campos EC. Binocularity in comitant strabismus: binocular visual fields studies. Doc Opbthalmol. 1982;53:249-281.

28. Jampolsky A. Characteristics of suppression in strabismus. Arch Opbthalmol. 1955;54:683-696.

29. Travers TàB. Suppression of vision in squint and its association with retinal correspondence and amblyopia. $\mathrm{Br} \mathrm{J} \mathrm{Ophthalmol.}$ 1938;22:577-604.

30. Schor C. Zero retinal image disparity: stimulus for suppression in small-angle strabismus. Doc Ophthalmol. 1978;46:149-160.

31. Schor CM. Visual stimuli for strabismic suppression. Perception $1977 ; 6: 583-593$.
32. Kilwinger S, Spekreijse H, Simonsz HJ. Strabismic suppression depends on the amount of dissimilarity between left- and right-eye images. Vis Res. 2002;42:2005-2011.

33. Ogle KN, Dyer JA. Some observations on intermittent exotropia. Arch Opbthalmol. 1965;73:58-73.

34. Campos EC, Chiesi C. Perimetrie binoculaire dans l'exotropie concomitant. Bull Soc Fr Opbthalmol. 1980;92:301-307.

35. Ramachandran VS, Cobb S, Levi L. The neural locus of binocular rivalry and monocular diplopia in intermittent exotropes. NeuroReport. 1994;5:1141-1144.

36. Jie Y, Xu ZY, He YN, et al. A 4 year retrospective survey of strabismus surgery in Tongren Eye Centre Beijing. Ophthalmic Physiol Opt. 2010; 30:310-314

37. Zhang ZL, Cantor CR, Schor CM. Perisaccadic stereo depth with zero retinal disparity. Curr Biol. 2010;20:1176-1181. 